\title{
BMJ Open Predictors of poor health and functional recovery following road trauma: protocol of a British Columbian inception cohort study
}

\author{
Leona K Shum, ${ }^{1}$ Herbert Chan, ${ }^{1,2}$ Shannon Erdelyi, ${ }^{1}$ Lulu X Pei (D) , ${ }^{1,3}$ \\ Jeffrey R Brubacher (i) ${ }^{1,2}$
}

To cite: Shum LK, Chan $\mathrm{H}$, Erdelyi S, et al. Predictors of poor health and functional recovery following road trauma: protocol of a British Columbian inception cohort study. BMJ Open 2021;11:e049623. doi:10.1136/ bmjopen-2021-049623

- Prepublication history and additional material for this paper is available online. To view these files, please visit the journal online (http://dx.doi.org/10. 1136/bmjopen-2021-049623).

Received 31 January 2021 Revised 18 March 2021 Accepted 23 March 2021

\section{Check for updates}

\section{Author(s) (or their} employer(s)) 2021. Re-use permitted under CC BY-NC. No commercial re-use. See rights and permissions. Published by BMJ.

${ }^{1}$ Department of Emergency Medicine, Faculty of Medicine, The University of British Columbia, Vancouver, British Columbia, Canada

${ }^{2}$ Centre for Clinical Epidemiology \& Evaluation (C2E2), Vancouver, British Columbia, Canada ${ }^{3}$ Department of Statistics, Faculty of Science, The University of British Columbia, Vancouver, British Columbia, Canada

Correspondence to Dr Jeffrey R Brubacher; jeff.brubacher@ubc.ca

\section{ABSTRACT}

Introduction Road trauma (RT) is a major public health problem affecting physical and mental health, and may result in prolonged absenteeism from work or study. It is important for healthcare providers to know which RT survivors are at risk of a poor outcome, and policy-makers should know the associated costs. Unfortunately, outcome after RT is poorly understood, especially for RT survivors who are treated and released from an emergency department (ED) without the need for hospital admission. Currently, there is almost no research on risk factors for a poor outcome among RT survivors. This study will use current Canadian data to address these knowledge gaps. Methods and analysis We will follow an inception cohort of 1500 RT survivors (16 years and older) who visited a participating ED within 24 hours of the accident. Baseline interviews determine pre-existing health and functional status, and other potential risk factors for a poor outcome. Follow-up interviews at 2, 4, 6, and 12 months (key stages of recovery) use standardised health-related quality of life tools to determine physical and mental health outcome, functional recovery, and healthcare resource use and lost productivity costs.

Ethics and dissemination The Road Trauma Outcome Study is approved by our institutional Research Ethics Board. This study aims to provide healthcare providers with knowledge on how quickly RT survivors recover from their injuries and who may be more likely to have a poor outcome. We anticipate that this information will be used to improve management of all road users following RT. Healthcare resource use and lost productivity costs will be collected to provide a better cost estimate of the effects of RT. This information can be used by policy-makers to make informed decisions on RT prevention programmes.

\section{INTRODUCTION}

In Canada, road trauma (RT) causes over 1900 fatalities and 150000 injuries annually, including 9000 serious injuries, ${ }^{1}$ with an annual cost estimated at CAD $\$ 37$ billion. ${ }^{2}$ Injury-related disability is a public health concern, ${ }^{3}{ }^{4}$ but outcome following RT and risk factors for a poor outcome are poorly studied, especially among cyclists, pedestrians
Strengths and limitations of this study

- Inception cohort design with large sample size $(n=1500)$.

- Measures self-reported health outcomes during key phases of injury recovery.

- Measures direct (healthcare) and indirect (lost productivity) costs.

- Risk of recall and reporting bias, especially for preinjury health status.

- Risk of sampling or non-respondent bias and/or bias from attrition.

and motorists involved in motor vehicle crashes with a 'minor injury' (emergency department (ED) visits without hospital admission). Outcomes are worse for RT survivors suffering severe injuries, but even 'minor injury' crashes can result in reduced healthrelated quality of life (HRQoL), including psychological harm (eg, Post-traumatic stress disorder (PTSD)) and prolonged work absenteeism (or inability to continue the same work prior to the crash due to new physical or psychological limitations). ${ }^{5}$ Psychological factors are important predictors of poor outcomes among RT survivors. ${ }^{6}$ Chronic pain is more common among RT survivors suffering from depression, anxiety, severe pain, multiple somatic complaints or PTSD in initial recovery stages. ${ }^{6-8}$ Other psychological contributors to chronic pain include health-seeking behaviour, poor recovery expectations, higher perceived collision severity, catastrophising and passive coping strategies. ${ }^{8-11}$ It is important to study injury outcomes among RT survivors (including all road users of all injury severity levels) and their associated risk factors, including baseline health status, socioeconomic and demographic factors, psychological factors and coping strategies. 


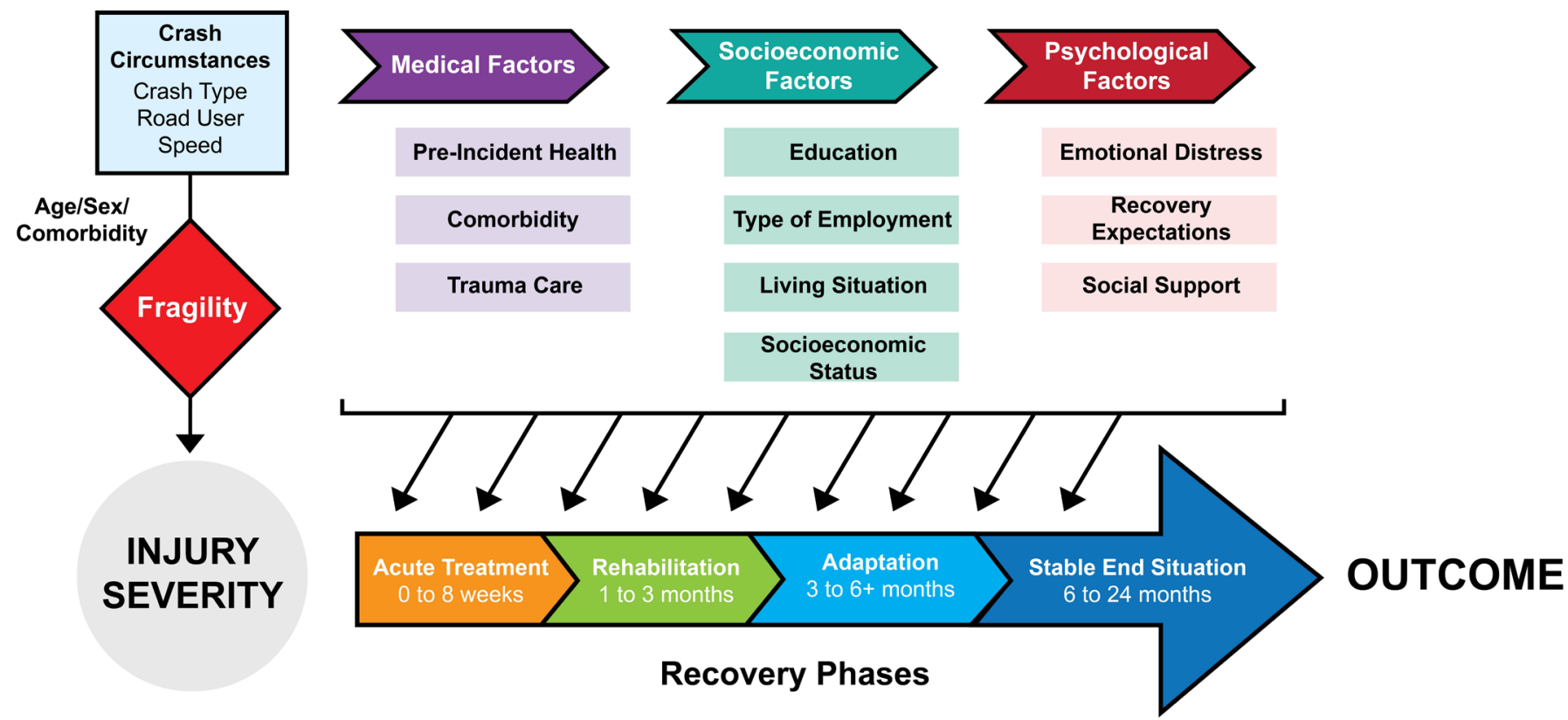

Figure 1 Factors affecting post-RT outcome. This conceptual diagram shows how potential risk factors act during different stages of recovery. Injury severity, a major determinant of outcome, depends on crash factors (transfer of kinetic energy) and the victim's fragility. Medical factors affect all stages of the recovery process. Socioeconomic factors impact both baseline health, ${ }^{13-15}$ and access to rehabilitation programmes or to resources that facilitate adaptation. ${ }^{16}$ Psychological factors may impact ability to comply with treatment or rehabilitation plans, or ability to adapt to injury-related disability. RT, road trauma.

The recovery trajectory for most injuries can be viewed as occurring during four phases (figure 1). ${ }^{12}$ The acute care phase (0-8 weeks) is characterised by intensive hospital management of injuries (eg, surgery). During rehabilitation (1-3 months), injured individuals develop increased capacity and move towards preinjury functioning. During adaptation (3-6 or more months), individuals modify their environment and personal routines to adapt to their limitations. Finally, in most cases, injured individuals reach a stable end situation within 6-12 months (rarely 24 months) of injury.

Potential risk factors influence the outcome during these recovery phases. Injury severity, a major determinant of outcome, is determined by circumstances of the injury event and the individual's fragility. Injury severity varies by road user type, age and sex. Medical factors affect all recovery phases. Socioeconomic factors impact both baseline health ${ }^{13-15}$ and subsequent recovery through access to rehabilitation programmes or resources facilitating adaptation. ${ }^{16}$ Psychological factors may impact an individual's ability to comply with treatment, follow rehabilitation plans, or adapt to injury-related disability. Clearly, it is important to follow participants throughout the four key recovery phases.

Previous RT research has methodological flaws limiting validity, generalisability and utility. One instance is use of retrospective cohorts of RT survivors who are enrolled after filing insurance claims, weeks following the crash, ${ }^{17} 18$ or after already developing symptoms as a result of the crash. ${ }^{19} 20$ Retrospective cohorts can result in selection bias if they exclude RT survivors who recover quickly from their injuries. Delayed enrolment can increase the likelihood of recall bias, especially related to preinjury HRQoL and accident details. Other RT research has limited generalisability as many studies excluded RT survivors involved in minor injury crashes, ${ }^{21}$ most excluded cyclists and pedestrians, ${ }^{92}$ and the majority excluded people with language barriers (non-native speakers). ${ }^{10} 23$ These are important gaps considering the increasing number of minor injury crashes in Canada, comprising the majority of RT cases, and their associated healthcare costs. ${ }^{24}$ Motor vehicle crashes involving cyclists and pedestrians will likely increase as active transportation (eg, cycling, walking) becomes more prevalent. ${ }^{25}$ Inclusion of non-native speakers may identify certain groups (eg, new immigrants) who may be at higher risk of RT and may experience worse outcomes. Additionally, many studies failed to conduct follow-up during key recovery phases, while others used idiosyncratic definitions or insurance company data to define outcomes. ${ }^{26}$ Many RT outcome studies have limited ability to identify risk factors for a poor outcome due to small sample sizes, ${ }^{27-29}$ not considering key risk factors (eg, psychological), or failure to identify or adjust for confounders like pre-existing health problems. ${ }^{21}$ Finally, current North American RT outcome research is limited as most studies have been conducted in Europe or Australia. These study design choices limit the impact of the research and ability to inform policy to improve outcomes of RT survivors.

Currently, research into risk factors for a poor outcome following RT is lacking, and methodological improvements are needed to address limitations 
of prior RT outcome research. The objective herein is to present the methodology of a multicentre study on the health and functional recovery of RT survivors who visited a participating ED in British Columbia (BC), Canada. This methodology addresses many limitations of current RT outcome research.

\section{METHODS AND ANALYSIS \\ Study design and setting}

This prospective observational study involves an inception cohort of RT survivors, with all injury severity levels. The study started recruitment in July 2018 and will run for 5 years. Participants are recruited from three BC EDs: Vancouver General Hospital (Vancouver), Royal Columbian Hospital (New Westminster) and Kelowna General Hospital (Kelowna). These hospitals serve rural, suburban and urban populations similar to those served by other trauma centres across Canada.

\section{Patient and public involvement}

The study was designed in consultation with public health stakeholders. Patients and/or general public were not involved in study design.

\section{Inclusion and exclusion criteria}

RT survivors (motorists, cyclists and pedestrians) aged 16 years and older who arrive in the ED within 24 hours of injuries sustained in a collision involving at least one motorised vehicle are included. Collisions not involving a motorised vehicle are excluded. Children younger than 16 years old are excluded as they have a different recovery trajectory and require different tools to measure HRQoL. Non-BC residents are also excluded as healthcare use during the recovery phase is not available for out-of-province participants. Cognitively impaired survivors are included if consent and study information could be obtained from a reliable proxy (eg, partner, parent). Non-English speakers are interviewed through a translator (eg, family) or multilingual research assistant (RA). RT survivors who are inappropriate to approach (suicidal, violent/aggressive, high alcohol or drug impairment, or in police custody) for the entire duration of their hospital visit or admission are excluded as reliable information cannot be obtained and it may be unsafe for research staff to approach the patient. For alcohol or drug impairment, individuals intoxicated on arrival at the ED, but subsequently sober during the same visit are included. Fatalities within 30 days following the hospital visit or admission are excluded.

\section{Recruitment}

Over 1.5 years of recruitment, it was estimated that 6600 RT survivors would be treated at participating EDs with at least 1200 severely injured patients admitted to hospital (figure 2). Given the large disproportion between minor (discharged home directly from the ED) and severely injured RT cases, all severely injured survivors and one-third representative sample of survivors with minor injuries are approached. A systematic sampling strategy is used to recruit a representative sample of RT survivors with minor injuries. RAs recruit participants from the ED for an average of 8 hours per day on a rotating schedule covering all times of day and days of the week (holidays included) throughout the year. Reasons for refusal to participate and failure to approach potential participants are recorded. The recruitment goal for the study is 1500 RT survivors (approximately 225 pedestrians, 300 cyclists and 975 motorists), including at least 750 who require hospital admission.

\section{Data sources and data management}

Data are collected from baseline interviews, medical records, follow-up interviews and administrative health records. Follow-up interviews at 2, 4, 6 and 12 months correspond to key phases of recovery: acute treatment, rehabilitation, adaptation and stable end situation ${ }^{12}$ (figure 1). We use the Research Electronic Data Capture online database for data management. ${ }^{30}$

\section{Baseline interviews}

Baseline interviews determine pre-existing health and functional status and other potential risk factors for a poor outcome. Baseline interviews are conducted in-person by RAs during ED visits or hospital admissions, or by telephone within 1-week postevent in some cases, to collect demographic and socioeconomic information, baseline health, crash/injury details and recovery expectations. Participants are approached as early as possible during their ED visit or hospital admission while respecting and prioritising their recovery. RT survivors who sustained severe injuries and are admitted to hospital are approached during their hospital admission. RT survivors with minor injuries are approached in the ED during their ED visit. All RT survivors are approached multiple times until a decision on participation in the study was obtained. RT survivors with minor injuries, who are discharged from the ED before they were able to decide whether to participate, are offered the opportunity to be consented and interviewed by telephone within 7 days of their ED visit.

The baseline interview (online supplemental appendix 1) includes the following domains: (1) crash details; (2) medical history (cardiorespiratory, neurological, gastrointestinal, musculoskeletal, psychiatric, other); (3) preevent anxiety and depression with the Patient Health Questionnaire-4 (PHQ) ${ }^{31} 32$; (4) somatic symptoms with the PHQ- $15^{33}$; and (5) pain catastrophising and coping with the Pain Catastrophising Scale. ${ }^{34}$ Baseline HRQoL is measured with the five-level EuroQol instrument (EQ-5D-5L-day before injury) and the Short Form 12 survey (SF-12-4 weeks prior to event). The EQ-5D-5L and SF-12 are validated tools assessing mental health (depression, anxiety), discomfort/pain, restrictions to bending or lifting, ambulation, self-care and daily and social activities. These tools have Canadian population norms and can 


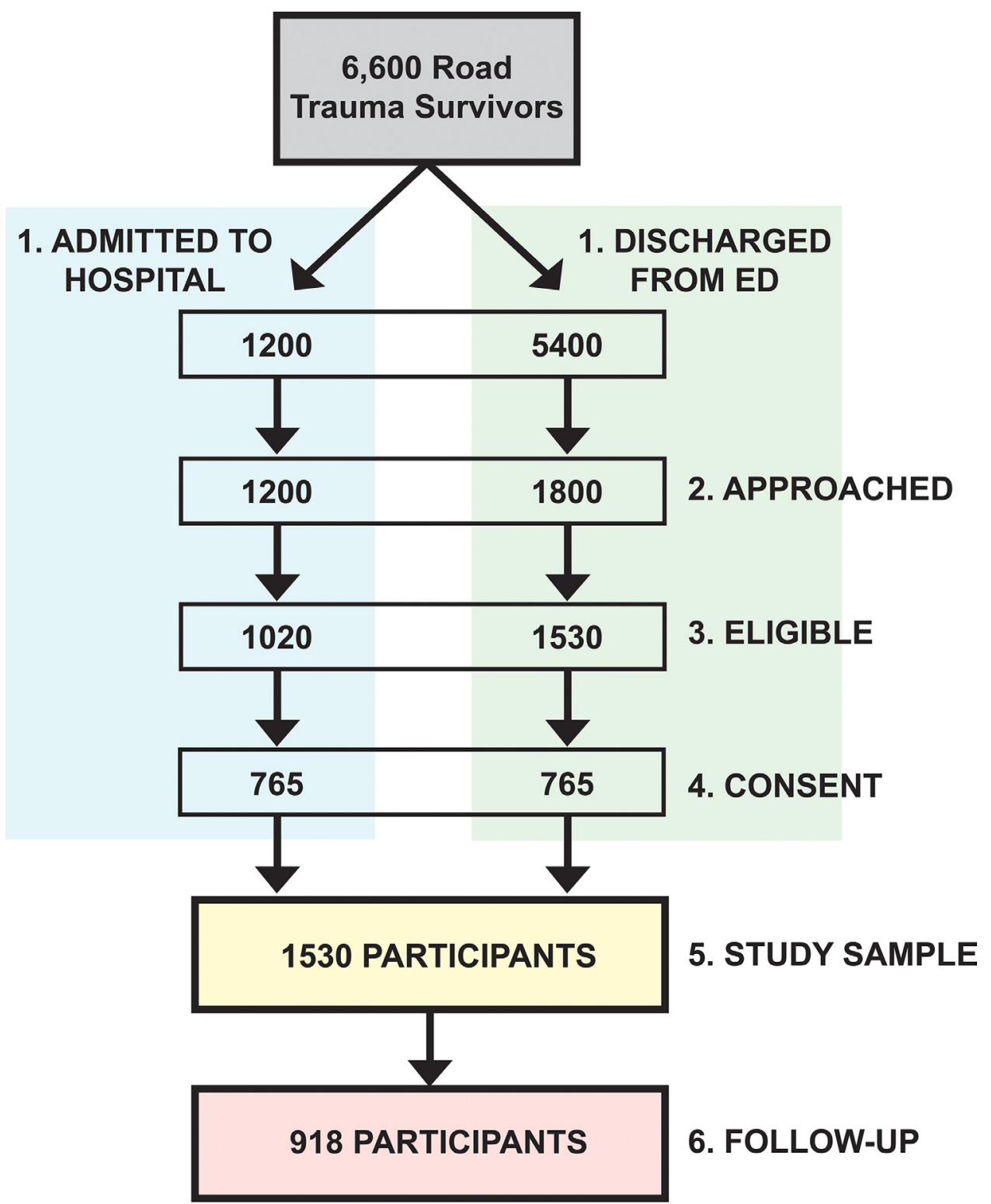

Figure 2 Recruitment flow chart. Anticipated recruitment and follow-up numbers over the duration of the study. This diagram illustrates estimated patient numbers for this study. Admitted to hospital and discharged from emergency department (ED): it was estimated that 4400 road trauma (RT) survivors per year would be treated at participating study sites. Of these, approximately $18 \%$ would be admitted to hospital and the rest would be discharged home directly from the ED. Approached: RAs (Research Assistants) approach all admitted RT survivors and use a systematic sampling strategy (based on the time of ED visit) to approach one-third of those who were discharged from the ED. Eligible: approximately $85 \%$ of RT survivors meet the inclusion and exclusion criteria. Consent: consent rates differ between those who were admitted to hospital and those who were discharged from the ED. It was estimated that $75 \%$ of admitted RT survivors and $50 \%$ of those discharged from the ED would consent to participate. Study sample: with these estimates, the recruitment goal of 1500 participants would be achieved within 2 years. Follow-up: the power calculations were based on a conservative $40 \%$ attrition rate (918 followed for 12 months). Attrition will not affect access to administrative data.

be used retrospectively to determine HRQoL. Preinjury productivity 4 weeks prior to the motor vehicle accident is assessed using the iMTA Productivity Cost Questionnaire (iPCQ). ${ }^{35}$ Participants are also asked about their expectations for recovery ('How long do you think it will take for you to fully recover from your injuries?').

\section{Follow-up interviews}

RT survivors' recovery trajectory and outcomes are assessed by follow-up interviews at 2, 4, 6 and
12 months postbaseline interview. Follow-up interviews (online supplemental appendix 2) include the EQ-5D-5L, SF-12, PHQ-15, Glasgow Outcome Scale (GOS-E), PTSD checklist (PCL-S) and iPCQ. The EQ-5D-5L and SF-12 are suitable for assessing individuals living independently whereas the GOS-E differentiates based on level of severe disability. The PCL-S is designed to detect PTSD following a traumatic event. ${ }^{36}$ The $\mathrm{iPCQ}$ is used to determine productivity 
losses related to absenteeism and reduced productivity at paid and unpaid work (eg, housework). Questions on recovery progress and return to daily activities are included. For example, participants are asked 'Have you fully recovered from the accident?' (options: 'yes', 'no', and 'don't know'). Self-reported healthcare utilisation (eg, physician visits, paramedical services) and quality of life difficulties (financial, legal, general) are also included in follow-up interviews.

Follow-up interviews are conducted by telephone, online survey, self-filled paper questionnaire or in-person depending on participant preference. For each follow-up interview, participants are contacted via telephone and email up to five times each. To maximise retention, more thorough and evidence-based retention strategies are applied including financial compensation and using alternate contact information (home, work and cell number, email, family or friend). Participants receive honorariums for completing the baseline $(\mathrm{CAD} \$ 15)$ and follow-up (CAD $\$ 10$ each) interviews. For those unable to complete interviews independently (eg, cognitive disability, language barrier), a proxy may either assist the participant or complete the questionnaire on the participant's behalf.

\section{Medical chart review}

Medical chart review of the index visit for all participants is the sole source of information for (1) injury type (eg, fracture) and location (eg, lower extremity); (2) injury severity $^{37}{ }^{38}$; (3) ED visit details (eg, arrival mode, acuity, duration, discharge diagnosis) and (4) ED investigations: diagnostic tests (eg, X-rays) and procedures (eg, sutures). Chart reviews are also used to supplement baseline interviews for information on: (1) accident details: road user type, location, single versus multiple-vehicle collision, seatbelt/helmet use; (2) medical history and (3) medication history. Medical charts of participating hospitals include ambulance run sheets which typically include accident details.

Standardised forms and protocols guide data extraction to ensure accuracy and consistency between RAs. A committee of experienced clinicians will review interview responses and medical charts to identify major discrepancies (eg, patient denies prior health problems, but medical record indicates hospital admissions) and arbitrate discrepancies (decide which data is most accurate). The number and type of major discrepancies will be reported and sensitivity analyses excluding those cases will be conducted.

\section{Administrative health records}

To measure healthcare resource use and calculate comorbidity scores, administrative health records including hospital admissions (Discharge Abstracts Database), medical service plan billings, ED visits (National Ambulatory Care Reporting System) and prescriptions (BC Pharmanet) are used. For participants who consent to Personal Health Number usage, records will be requested through PopDataBC, a health data depository supporting research with access to individual-level, de-identified longitudinal data on BC residents. ${ }^{39}$ Data will be collected for 1 year prior to and 1 year following the crash to compare healthcare resource use preaccident and postaccident. Healthcare services not covered by public health insurance will be identified during the follow-up interviews.

\section{Analysis}

The following dichotomous outcomes will be assessed: (1) self-reported incomplete recovery; (2) reduction from baseline 'pre-event' values on EQ-5D-5L, SF-12 and PHQ-15 exceeding minimal clinically important difference values reported for these scales; (3) evidence of PTSD and (4) have not returned to work, school, or usual activities. At each follow-up period, the percentage of participants who experience each of the above poor outcomes will be reported. Descriptive statistics will be generated for all study participants, disaggregated by sex, age group, socioeconomic factors, road user type, and disposition (discharged from ED or admitted to hospital).

The following candidate risk factor categories will be examined: (1) demographic and socioeconomic variables (sex, age, ethnicity, residence location, marital status, employment status and education level); (2) baseline health status (preinjury SF-12 and EQ-5D-5L scores, chronic disease score, self-reported medical history, previous year hospital admissions and physician visits); (3) psychosocial factors (anxiety, depression and catastrophising/coping); (4) injury type, location and severity and (5) road user type (pedestrian, cyclist and motorist) and accident details.

For outcomes 1-4 defined above, separate mixed effects log-binomial regression models (generalised linear mixed models (GLMMs) using log link function), will be fitted to estimate relative risks (RRs) and confidence intervals (CIs) for associations between risk factors and poor outcomes measured at 2, 4, 6 and 12 months. The nested structure of the data will be accounted for by including a random intercept for hospital site and participants nested within each site. Since GLMMs can be unstable in the presence of many predictors, separate models for each risk factor to obtain unadjusted RRs for poor outcome will be fitted first. These models will also include follow-up period (2, 4, 6 or 12 months) as a categorical predictor and an interaction term between period and risk factor. This will allow estimation of recovery trajectories and risk factor impact at different recovery stages. Next, a single model to identify independent predictors of outcome and estimate adjusted RRs will be built. This model will include multiple candidate risk factors identified using Harrell's approach. ${ }^{40}$ A L1-penalised estimation will also be used as this method combines shrinkage with variable selection for GLMMs and works well when there are many influential predictors. ${ }^{41}$ A Bonferroni-adjusted significance level will be used. 


\section{Missing data}

The percentage of participants with missing baseline data is expected to be $<4 \%$ based on pilot research. Assuming missing data are not related to the outcome, no bias will result from excluding these subjects. ${ }^{42}$ For partially complete follow-up interview responses, guidelines of each validated tool will be followed to obtain on outcome score. As a mixed-effects log-binomial regression model is proposed, missing response data for participants who are lost to follow-up will be ignored. GLMMs use all available data and provide unbiased estimates if data are missing at random (unobserved data depend only on observed data). Further statistical testing using t-tests for continuous risk factors and chi-squared tests for categorical risk factors will be performed to explore differences between RT survivors who complete the study and those who are lost to follow-up.

\section{Sample size considerations}

Sample size calculation is for outcome data at 12 months and conducted for three road user types (pedestrians, cyclists and motorists). A conservative $40 \%$ attrition is assumed such that 12-month outcome data will be available for at least 135 pedestrians, 180 cyclists and 585 motorists. With an estimated prevalence of $35 \%$ for outcomes and $50 \%$ for risk factors, and using a significance level of 0.0125 corrected for multiple outcomes, this study will have $80 \%$ power to detect RRs of 2.3, 2.0, and 1.5 for pedestrians, cyclists and motorists, respectively. These estimates are based on two-sided comparison of independent proportions using the Normal approximation described by Woodward. ${ }^{43}$

\section{Healthcare resource use}

A total healthcare cost will be obtained for every study participant, supplemented by lost productivity costs. Generalised linear models will be fit to explore variation in healthcare and lost productivity costs according to road user type, injury severity, age range, sex and disposition. Study participants will be differentiated by those who complete follow-ups and those who are lost to follow-up with respect to baseline characteristics.

\section{ETHICS AND DISSEMINATION \\ Ethics approval}

This study is approved by the research ethics board of the University of British (approval certificate number: H18-00284) and by research ethics boards for the other participating study sites: Fraser Health Authority (New Westminster, BC) and Interior Health Authority (Kelowna, BC). Note that there is a harmonised ethics review process for $\mathrm{BC}$ sites. Ethics approval is renewed annually and updated throughout the duration of the study.

Participants provide informed written or verbal consent. For minors (16-18 years old), parental/guardian permission is obtained in addition to participant assent. For participants unable to provide consent (eg, comatose), proxy consent is obtained from a designated caregiver.

\section{Importance of this research}

The Road Trauma Outcome Study (RTOS) is designed to overcome many limitations of previous RT outcome research. It uses a robust methodology that will add to the RT outcome knowledge base. First, it recruits an inception cohort of RT survivors during their ED visit (or hospital admission) following a crash. Inception cohorts are ideal for studying outcome and prognostic factors and are less prone to sampling bias compared with retrospective cohorts. ${ }^{44}$ To maximise generalisability, recruited RT survivors include: all road user types with all injury severity levels; non-native speakers (using translators); and those with cognitive limitations (with history obtained from caregivers). Another strength is the use of patient-reported outcomes to study the effects of injury on daily lives of RT survivors; this study uses validated standardised tools to study HRQoL from physical and psychological domains during key recovery phases. ${ }^{412}$ This study includes a large sample size, determines healthcare costs associated with RT, and includes productivity loss estimates at work and home. The sample of 1500 RT survivors provides sufficient power to study key risk factors for a poor outcome. It is also important to study RT outcome in North America as many risk factors for poor recovery, including recovery expectations and crash severity perception, ${ }^{10}$ are likely related to cultural factors that vary between countries.

Total economic cost attributed to an injury is a combination of direct costs (healthcare costs from injuries) and indirect costs (due to reduced productivity from hospitalisation, disability and premature death). ${ }^{4647}$ This study will determine healthcare and lost productivity costs for RT survivors, providing a more accurate and complete economic assessment and subsequently informing policy towards improving health delivery programmes. Several instruments measure productivity loss; we used the iPCQ as it has been tested in the general population. ${ }^{35}$ Moreover, the iPCQ allows for separate quantification of productivity losses due to absenteeism, presenteeism and unpaid work. ${ }^{35} 48$ The value of time lost from work and homemaking due to injury is measured by earnings data and market value of unperformed homemaking services, respectively. ${ }^{46}$ This study addresses knowledge gaps including health and financial consequences, productivity impacts and risk factors for a poor outcome following RT.

\section{Limitations of study design}

Although our study design improves on previous research, it still has limitations which have been addressed as best as possible to minimise their effects. These limitations include recall and reporting bias from using self-reported standardised tools, especially related to precrash health. The 'good-old-days' bias, where patients knowingly or unknowingly exaggerate 
their preinjury HRQoL, is common following injury. ${ }^{49-51}$ To minimise 'good-old-days' and recall bias, baseline interviews were conducted as soon as possible following the crash, ideally within 7 days. ${ }^{12}$ Administrative health records, including calculated chronic disease score, will be used as an objective measure of preinjury health. ${ }^{49}$ Participants are assured their responses are confidential, and identifying as health researchers strengthens rapport and improves response rate and quality. ${ }^{52}$ Another limitation is sampling bias or non-respondent bias which may occur if those who are missed or decline to participate differ in important ways from participants. Refusals are tracked and differences between participants and those who refused to participate with respect to age, sex, road user type, and hospital admission required will be reported. Additionally, using modest honorariums and assurance of confidentiality is intended to minimise refusals, and the analysis plan also considers non-response bias. Inherently, our study cannot be generalised to RT survivors who never seek medical care in a hospital setting or seek care days later. Finally, attrition may affect the study findings in terms of overall response rate and baseline characteristics of those who complete follow-ups compared with those lost to follow-up. Different contact methods are used to minimise attrition rate. These strategies to minimise bias and missing data are applied during recruitment and analysis to help reduce the effects of these limitations.

\section{Expected outcomes and benefits}

This research will advance understanding of the impact of RT on individuals treated in hospital for RT injuries. It will identify risk factors for poor outcomes and provide better estimates of direct and indirect RT costs. These findings are relevant to RT survivors and their families, healthcare providers, public health officials, healthcare and traffic policy makers and researchers. Understanding recovery trajectory and risk factors for a poor outcome following RT may inform the development of rehabilitation programmes and help clinicians identify RT survivors who would benefit from more intensive care, possibly earlier in their recovery trajectory. These findings may also help RT survivors and their families set expectations for recovery, possibly reducing the adverse psychological consequences commonly experienced by RT survivors. This research will also provide a better understanding of the impact of RT on healthcare costs and productivity and provide data and tools that other researchers can use for future economic analyses of RT prevention programmes. Traffic policy-makers and public health officials may use these cost estimates to make better decisions about allocating limited resources for expensive RT prevention programmes. Thus, these findings will have practical implications for RT survivors and their families, healthcare providers, policy-makers, public health officials and other researchers.

\section{SUMMARY}

The RTOS is a large inception cohort study that will provide a comprehensive description of outcome after RT including motor vehicle crashes of all severity levels for all road users, identify risk factors for poor outcomes, and determine direct healthcare and lost productivity costs associated with RT. This information can be used by numerous stakeholders who have an interest in preventing RT or improving outcome for RT survivors.

Contributors This research study methodology was conceptualised and developed by JRB (principal investigator) and HC. SE and LXP assisted with the data analysis plans. LKS drafted the manuscript and is coordinating the study. All authors reviewed and approved the final manuscript.

Funding This research was supported by a Canadian Institutes of Health Research (ClHR) Project Grant (application number 388776).

Disclaimer The funding organisations had no role in project design or implementation. The funding organisations placed no restrictions on the publication of findings from this research.

Competing interests None declared.

Patient consent for publication Not required.

Provenance and peer review Not commissioned; externally peer reviewed.

Supplemental material This content has been supplied by the author(s). It has not been vetted by BMJ Publishing Group Limited (BMJ) and may not have been peer-reviewed. Any opinions or recommendations discussed are solely those of the author(s) and are not endorsed by BMJ. BMJ disclaims all liability and responsibility arising from any reliance placed on the content. Where the content includes any translated material, BMJ does not warrant the accuracy and reliability of the translations (including but not limited to local regulations, clinical guidelines, terminology, drug names and drug dosages), and is not responsible for any error and/or omissions arising from translation and adaptation or otherwise.

Open access This is an open access article distributed in accordance with the Creative Commons Attribution Non Commercial (CC BY-NC 4.0) license, which permits others to distribute, remix, adapt, build upon this work non-commercially, and license their derivative works on different terms, provided the original work is properly cited, appropriate credit is given, any changes made indicated, and the use is non-commercial. See: http://creativecommons.org/licenses/by-nc/4.0/.

\section{ORCID iDs}

Lulu X Pei http://orcid.org/0000-0002-1168-7990

Jeffrey R Brubacher http://orcid.org/0000-0002-4866-4231

\section{REFERENCES}

1 Statistics Canada. Canadian motor vehicle traffic collision statistics: 2018, 2019. Available: https://www.tc.gc.ca/eng/motorvehiclesafety/ canadian-motor-vehicle-traffic-collision-statistics-2018.html

2 Canadian Council of Motor Transport Administrators. Towards zero: the safest roads in the world. 2016 Jan.

3 Newgard CD. Long-Term global health deficits: yet another adverse outcome from motor vehicle crashes. Ann Emerg Med 2006;48:737-8.

4 Polinder S, Haagsma JA, Belt E, et al. A systematic review of studies measuring health-related quality of life of general injury populations. BMC Public Health 2010;10:1-13.

5 Hours M, Chossegros L, Charnay P, et al. Outcomes one year after a road accident: results from the ESPARR cohort. Accid Anal Prev 2013;50:92-102.

6 Wideman TH, Sullivan MJL. Development of a cumulative psychosocial factor index for problematic recovery following workrelated musculoskeletal injuries. Phys Ther 2012;92:58-68.

7 McLean SA, Ulirsch JC, Slade GD, et al. Incidence and predictors of neck and widespread pain after motor vehicle collision among US litigants and nonlitigants. Pain 2014;155:309-21.

8 Carroll LJ, Holm LW, Hogg-Johnson S, et al. Course and prognostic factors for neck pain in whiplash-associated disorders (WAD): results of the bone and joint decade 2000-2010 Task force on neck pain and its associated disorders. J Manipulative Physiol Ther 2009;32:S97-107. 
9 Wynne-Jones G, Jones GT, Wiles NJ, et al. Predicting new onset of widespread pain following a motor vehicle collision. J Rheumatol 2006;33:968-74.

10 Carroll LJ, Holm LW, Ferrari R, et al. Recovery in whiplashassociated disorders: do you get what you expect? J Rheumatol 2009;36:1063-70.

11 Wideman TH, Sullivan MJL. Differential predictors of the longterm levels of pain intensity, work disability, healthcare use, and medication use in a sample of workers' compensation claimants. Pain 2011;152:376-83.

12 Van Beeck EF, Larsen CF, Lyons RA, et al. Guidelines for the conduction of follow-up studies measuring injury-related disability. $J$ Trauma 2007;62:534-50.

13 Adler NE, Ostrove JM. Socioeconomic status and health: what we know and what we don't. Ann N Y Acad Sci 1999;896:3-15.

14 Berkman L, Epstein AM. Beyond health care - socioeconomic status and health. New England Journal of Medicine 2008;358:2509-10.

15 Mackenbach JP, Stirbu I, Roskam A-JR, et al. Socioeconomic inequalities in health in 22 European countries. New England Journal of Medicine 2008;358:2468-81.

16 Adler NE, Newman K. Socioeconomic disparities in health: pathways and policies. Health Aff 2002;21:60-76.

17 Ozegovic D, Carroll LJ, Cassidy JD. Factors associated with recovery expectations following vehicle collision: a population-based study. J Rehabil Med 2010;42:66-73.

18 Gopinath B, Jagnoor J, Harris IA, et al. Prognostic indicators of social outcomes in persons who sustained an injury in a road traffic crash. Injury 2015;46:909-17.

19 Pobereskin LH. Whiplash following rear end collisions: a prospective cohort study. J Neurol Neurosurg Psychiatry 2005;76:1146-51.

20 Bunketorp L, Stener-Victorin E, Carlsson J. Neck pain and disability following motor vehicle accidents--a cohort study. Eur Spine J 2005;14:84-9.

21 Ameratunga SN, Norton RN, Bennett DA, et al. Risk of disability due to car crashes: a review of the literature and methodological issues. Injury 2004;35:1116-27.

22 Ameratunga SN, Norton RN, Connor JL, et al. A population-based cohort study of longer-term changes in health of car drivers involved in serious crashes. Ann Emerg Med 2006;48:729-36.

23 Gopinath B, Jagnoor J, Nicholas M, et al. Presence and predictors of persistent pain among persons who sustained an injury in a road traffic crash. Eur J Pain 2015;19:1111-8.

24 Columbia ICoB. Rate pressures 2017, 2017. Available: https://www. icbc.com/about-icbc/newsroom/Pages/rate-pressures-claimscrashes-costs.aspx

25 EcoPlan I. Active transportation in Canada: a resource and planning guide. Ottawa, Ontario, Canada: Transport Canada, Environmental Initiatives Group, 2011.

26 Gustafsson M, Stigson H, Krafft M, et al. Risk of permanent medical impairment (RPMI) in car crashes correlated to age and gender. Traffic Inj Prev 2015;16:353-61.

27 Brooke KJ, Faux SG, Wilson SF, et al. Outcomes of motor vehicle crashes with fracture: a pilot study of early rehabilitation interventions. J Rehabil Med 2014;46:335-40.

28 Littleton SM, Hughes DC, Poustie SJ, et al. The influence of fault on health in the immediate post-crash period following road traffic crashes. Injury 2012;43:1586-92.

29 Ottosson C, Pettersson H, Bergman B, et al. Personality disorders are not associated with nonrecovery in patients with traffic-related minor musculoskeletal injuries. Journal of Trauma-Injury Infection Critical Care 2010;68:198-203.

30 Harris PA, Taylor R, Thielke R, et al. Research electronic data capture (REDCap)--a metadata-driven methodology and workflow process for providing translational research informatics support. J Biomed Inform 2009;42:377-81.

31 Löwe B, Wahl I, Rose M, et al. A 4-item measure of depression and anxiety: validation and standardization of the patient health Questionnaire-4 (PHQ-4) in the general population. J Affect Disord 2010;122:86-95.

32 Kroenke K, Spitzer RL, Williams JBW, et al. An ultra-brief screening scale for anxiety and depression: the PHQ-4. Psychosomatics 2009;50:613-21.

33 Kocalevent R-D, Hinz A, Brähler E. Standardization of a screening instrument (PHQ-15) for somatization syndromes in the general population. BMC Psychiatry 2013;13:91.

34 Sullivan MJL, Bishop SR, Pivik J. The pain catastrophizing scale: development and validation. Psychol Assess 1995;7:524-32.

35 Bouwmans $\mathrm{C}$, Krol M, Severens $\mathrm{H}$, et al. The iMTA productivity cost questionnaire: a standardized instrument for measuring and Valuing health-related productivity losses. Value Health 2015;18:753-8.

36 Wilkins KC, Lang AJ, Norman SB. Synthesis of the psychometric properties of the PTSD checklist (PCL) military, civilian, and specific versions. Depress Anxiety 2011;28:596-606.

37 Osler T, Rutledge R, Deis J, et al. ICISS: an international classification of disease-9 based injury severity score. J Trauma 1996;41:380-6.

38 Barnard RT, Loftis KL, Martin RS, et al. Development of a robust mapping between AIS 2+ and ICD-9 injury codes. Accid Anal Prev 2013;52:133-43.

39 Populationdata ${ }^{\mathrm{BC}}$. Population data bc, December 2020. Available: www.popdata.bc.ca

40 Harrell F. Regression modeling strategies with application to linear models, logistic regression and survival analysis. New York, NY: Springer-Verlag, 2001.

41 Groll A, Tutz G. Variable selection for generalized linear mixed models by L 1-penalized estimation. Stat Comput 2014;24:137-54.

42 Sterne JAC, White IR, Carlin JB, et al. Multiple imputation for missing data in epidemiological and clinical research: potential and pitfalls. BMJ 2009;338:b2393

43 Woodward M. Formulae for sample size, power and minimum detectable relative risk in medical studies. The Statistician 1992;41:185-96.

44 Anonymous. How to review the evidence: systematic identification and review of the scientific literature. Canberra, Australia: National Health and Medical Research Council, 1999.

45 Mann CJ. Observational research methods. research design II: cohort, cross sectional, and case-control studies. Emerg Med $J$ 2003;20:54-60.

46 Parachute. The cost of injury in Canada. Toronto, Ontario: Parachute, 2015.

47 Polinder S, Haagsma J, Panneman M, et al. The economic burden of injury: health care and productivity costs of injuries in the Netherlands. Accid Anal Prev 2016;93:92-100.

$48 \mathrm{Krol} \mathrm{M}$, Brouwer W. How to estimate productivity costs in economic evaluations. Pharmacoeconomics 2014;32:335-44.

49 Satya-Murti S, Lockhart J. Recognizing and reducing cognitive bias in clinical and forensic neurology. Neurol Clin Pract 2015;5:389-96.

50 Scholten AC, Haagsma JA, Steyerberg EW, et al. Assessment of preinjury health-related quality of life: a systematic review. Popul Health Metr 2017;15:1-10.

51 Don AS, Carragee EJ. Is the self-reported history accurate in patients with persistent axial pain after a motor vehicle accident? Spine $J$ 2009;9:4-12

52 Cassell J, Miller P. Is it self-administration if the computer gives you encouraging looks? In: Conrad FG, Schober MF, eds. Envisioning the survey interview of the future. Hoboken, New Jersey: John Wiley \& Sons, Inc, 2007: 161-78. 